\title{
Business Aspects of the Neutral Host Model: The Immersive Video Services Case
}

\author{
Ioannis Neokosmidis ${ }^{1(\bowtie)}$, Vangelis Logothetis ${ }^{1}$, Theodoros Rokkas ${ }^{1}$, \\ Luca Vignaroli $^{2}$, Davide Desirello ${ }^{2}$, Antonino Albanese ${ }^{3}$, \\ Viscardo Costa $^{3}$, Mariano Lamarca ${ }^{4}$, Maria Rita Spada ${ }^{5}$, \\ Muhammad Shuaib Siddqui ${ }^{6}$, Dimitra Simeonidou ${ }^{7}$, \\ and Carlos Colman-Meixner ${ }^{7}$ \\ 1 inCITES Consulting, Strassen, Luxembourg \\ i.neokosmidis@incites.eu \\ 2 RAI, Centro Ricerche, Innovazione Tecnologica e Sperimentazione, \\ Turin, Italy \\ 3 ITALTEL, Castelletto, Milan, Italy \\ ${ }^{4}$ IMI - Barcelona City Council, Barcelona, Spain \\ 5 Wind Tre S.p.A, Largo Metropolitana 5, Rho, Milan, Italy \\ 6 i2CAT Foundation, Barcelona, Spain \\ ${ }^{7}$ High Performance Laboratory, University of Bristol, Bristol, UK
}

\begin{abstract}
The introduction of novel approaches that cause a paradigm shift on a technological level can become the driver of innovative business models that benefit from capabilities that may have emerged. Such is the case for $5 \mathrm{G}$ which offers an entirely new way of delivering services that affects consumers and even more fundamentally the providers. The introduction of network slicing and the ability to provide individually configurable parts of the network to providers offer unique opportunities and solutions particularly when it comes to solving the known issue of overlapping infrastructure investments from various stakeholders. In this paper, the scenario of a single wholesale $5 \mathrm{G}$ network provider the Neutral Host - operating in the City of Lucca is examined. Some remarks about the impact of the $\mathrm{NH}$ model on Barcelona superblock urbanistic model are also provided.
\end{abstract}

Keywords: Neutral Host $\cdot 5 \mathrm{G} \cdot$ Business model $\cdot$ Technoeconomics

\section{Introduction}

With the emergence of mobile communication networks, a great variety of services have been introduced to consumers along with the relevant revenue streams that emerged for providers of all sorts. With bandwidth demands following a steady increase, the necessity for updating the infrastructure and accommodate the observed trends is emerging. The technology of $5 \mathrm{G}$ aims to do just that by providing far greater internet speeds compared to its predecessors. But talking about increased connection speeds is only scratching the surface of what $5 \mathrm{G}$ can offer to the telecommunications world since great innovations are adopted into the network's architecture on a foundational level. 
In particular, the ability to create network slices creates unique business opportunities as network sharing could be efficient and very adjustable to different service providers' needs, something that was not the case up until this point. Furthermore, 5G envisions beyond conventional passive networking sharing and encompass active network sharing as well. Based on this major characteristic, the concept of the Neutral Host (NH) becomes more appealing since it provides the foundations that allow the seamless development of a single entity that can build virtual end-to-end slices including various resources (e.g. network storage, computing) that can be leased to mobile network operators that operate these resources per their respective needs.

Thus, the NH model allows for the deployment of a single network capable of hosting various providers reducing infrastructure competition and leading to an overall welfare increase. In the case of urban areas, the deployment of small cells over street furniture will be more intensive while physical coexistence of multiple operators become a Chimera. Even though the NH concept has existed beforehand, the ability that $5 \mathrm{G}$ has to parameterize network slices and adhere to providers' needs, is what makes it able to crossover from a theoretical model to an actual implementation that shows promise in overcoming challenges faced by infrastructure providers and vertical actors $[1,2]$. Being able to create networks that can be leveraged upon multiple vendors renders the $\mathrm{NH}$ model capable to support the fast adoption and exploitation of $5 \mathrm{G}$ as there is no longer the need to develop multiple isolated access networks to facilitate services. Apart from the technological aspects that characterize the $\mathrm{NH}$, there also exists the business perspective that surfaces alongside an entity that can potentially be the arbitrator for a whole new array of SLAs that will characterize the network [2-4].

A business model encompassing the characteristics of the described $\mathrm{NH}$ is presented followed by a technoeconomic analysis that aims to explore the model's potential gauged in economic terms. Increased flexibility is the main attribute of the modelled wholesaler, not only in terms of services provided, but also when it comes to charging strategies that can be diversified in creative ways. Typical wholesale access pricing is still present but can be shaped in more suitable ways to better suit each hosted entity. Dynamic monthly fees could be the case or even a pay-per-use scheme if periodic access to the infrastructure is needed, as would be the case for some content providers that would only employ the network's capabilities during specific timeframes, for example during Public events. The benefit to this approach is the ability to charge based on actual resources utilized on top of a flat access rate, instead of only the later which is usually encountered in the industry and bound to be pricier if it acts as a universal price for all possible services. This perspective combined with the network slicing will provide additional flexibility as the $\mathrm{NH}$ can dynamically adjust to the needs of various interested parties. Additional specialization can be achieved by adopting perservice pricing further increasing possible revenues.

It should be highlighted that Network SLAs stability by compromising coverage and signal to noise ration (SNR), should also be taken into account. 5GCity project's deployments in both Lucca and Barcelona show that it is possible to provide a flat coverage higher than that of the $4 \mathrm{G}$ or WIFI model with less transmission power and higher SNR managing adequately distance and height of SCs. That means QoS guarantee independently of phones' position in the coverage area of the SC. 
The rest of the paper is organized as follows: Sect. 2 describes the Neutral Host business model. Section 3 discusses the technoeconomic analysis of the NH model. Section 4 discusses and concludes the paper.

\section{The Neutral Host Business Model}

The telecommunication industry has undoubtedly seen a lot of growth in recent years as connectivity became a staple of everyday life and demand for capacity, both residential and business, steadily increased without showing any signs of slowing down. Regardless of this continued increase in consumption and reliance on ICT and digital services, operators' profits have suffered underlying the need to identify new ways of profiting from infrastructure. Bearing that in mind and understanding the importance of context, a Business Model Canvas methodology is being used to identify the ecosystem that revolves around the $\mathrm{NH}$ along the potential opportunities that exist. This methodology looks into external and internal aspects that need to be assessed when developing a business model.

\subsection{External Aspects}

Initially, the business model examines the external environment of the NH. The proposed idea is for the NH to offer a platform that combines infrastructure and service dashboards. The former allows mobile network operators to deploy network slices in a wholesale manner. Although, operators are interested in 5G, they appear reluctant to invest because $5 \mathrm{G}$ antennas size and range ask for much denser deployments that create practical backhauling and powering issues. Additionally, the $\mathrm{NH}$ offers, through the service dashboard, access to a service design toolkit (SDK) that allows the further exploitation of 5G's capabilities. Vertical application developers can use it to develop new services and networks functions while service providers can combine available functions and create new services. Such an approach would allow for greater flexibility as a result of the combination of software-based functionalities along with physical access. As such, the reliance on wholesale access is relaxed and the $\mathrm{NH}$ can look into entirely new revenue streams and most importantly explore the untapped potential of fluid business models that still benefit from the established ways of exploiting network infrastructure. Moreover, RAN architectures can be innovated with multi-RAT function disaggregation, implemented through SDN-based virtual RAN slicing and RAN function virtualization for LTE and Wi-Fi. Also, the NH can support edge computing capabilities to enable ultra-low latency, high bandwidth and real-time access to radio network information that can be leveraged by applications. All these characteristics render the $\mathrm{NH}$ model an enticing option with significant added value.

The NH would be able to reach out to its clients to communicate the mentioned benefits of its business model through direct sales or dealers establishing a clear relationship through the use of online support, call centers and in field assistance.

As the services that can be provided are described, it is crucial to identify the possible revenue streams for the NH's business model. Wholesale access to the infrastructure is the obvious one but with added ability to adopt new pricing schemes 
such as monthly subscription or even more dynamic choices (e.g. pay-as-you-go, per core etc.) which would substantiate the use of two-part tariffs. Opting for a basic network slice pricing, based on specific KPIs (e.g. maximum throughput, number of users connected or maximum/average latency) which can be adapted based on actual resource usage. This approach would not only incentivize mobile network operators to utilize the Neutral Host's network but also help in keeping up with overall resource usage estimation, opening up the network for better management and exploitation.

Similarly, the platform offering the services development toolkit can be subject to different pricing schemes such as subscription fees or fee per package of $\mathrm{N}$ services. Alternatively, a more dynamic scheme could be offered such as charging per service or per number of services and/or per function used for the creation of the new service. This platform, based on the combination of cloud and edge computing and the SDK for service creation, will be able to provide pricing flexibility in order to service providers and OTTs that will need to utilize parts of the network in ways suitable to the products to be delivered.

Another revenue stream that is important to capture is that of private networks that is considered a critical aspect of the potential of $5 \mathrm{G}$ networks. A company will be able to utilize infrastructure and service platform to host its own bundle of services for their employees/users and evolution to the "private LTE" networks currently available that is also usable in "mission critical" scenarios. To offer this type of services, the allocation of specific spectrum is needed regardless of where it comes from (the industry itself or a MNO). Finally, the NH can profit from the provision of certifications to developers that intend to certify their services uploaded to the catalogue and from consulting and training services.

\subsection{Internal Aspects}

Apart from the exterior, an internal analysis is also conducted to understand the aspects that deal with the actions that the $\mathrm{NH}$ has to perform as well as the resources that need to be allocated in order to successfully develop and maintain the infrastructure and platform. The key resources used for the proper execution of the business model are the business and technical personnel, the physical assets such as network equipment, and the facilities that need to be made available, e.g. sites for network equipment, lampposts, street signs, traffic lights etc. In a City, it is important to have a scalable model for the development of an end to end solution allowing $\mathrm{NH}$ to provide same services with same QoS independently of the density of the deployed SCs.

The Neutral Host, apart from building the network is also tasked with its operation and the provisioning of network slices and required services and functions to the hosted entities. Also, an initial collection of available services is a task that may need to be carried out by the NH in order to establish the basic functionalities that can then be enhanced by developers certified by the host himself. Training and consulting should also be considered as entirely new types of functionalities are being proposed.

A crucial component of the project's fruition would be to establish key partnerships with NFV providers and software developers that would provide VNFs, NFI and orchestrators. Partnership with vendors shows a lot of potential as technologies developed by for the $\mathrm{NH}$ can be integrated and sold by them in other network implementations. 
Apart from business partners, cooperation with the open source and scientific community can also reveal pathways to innovation. Equally important is the ability to come together with facility managers and landlords as without the necessary space that they can offer the infrastructure cannot be realized.

As is true for every network implementation though, the $\mathrm{NH}$ needs to allocate significant monetary resources for the deployment and operation of the $5 \mathrm{G}$ network. Capital and operational expenditure to build and run the network but also the costs related to the integration of the platform that lies at the heart of the business models. Also, not to be overlooked is the research and development that would need to come before during and after the development of the network to create, integrate and keep in line with technological advancements.

\section{Technoeconomic Analysis}

To explore the economic side of this model, a technoeconomic (TE) analysis is conducted. The use case under investigation deals with a NH providing wholesale access to MNOs that offer immersive video services (IVS). It should be highlighted that these services are designed to allow end-users to move through parts of the city utilizing VR/AR/MR-like devices and receiving content related to the surrounding environments. This experience is enhanced using additional content in the form of $360^{\circ}$ video, further increasing immersion and information delivered to the end user. Another interesting feature examined is the visual search that allows visual content captured by the user (e.g. such as buildings, statues, paintings) to be matched to content present in databases thanks to visual similarities and provide information, for example, looking at the Parthenon could allow the user to examine painted graphical representations of how scientists believe the monument looked like in the past.

Television archives in the form of 2D video, panoramic video and 3D models as well as new UHD/4K content will be available to the users, creating an all-around digital experience. To successfully achieve that, ultra-high bandwidth and very low system latency are required in order to effectively provide the described content to endusers. The services will leverage the NH model that allows the creation of end-to-end segmented slices, which encompass a wide variety of resources. The slices are leased to MNOs, which can then operate their virtual resources by mapping their services with respect to the set of slices that they have been assigned.

The TE analysis, particularly focuses on three main services: (a) on demand immersive tour experience using Oculus [5] headgear in the context of Puccini Museum [6], (b) $360^{\circ}$ live content streaming during crowded events, with reference to Piazza Napoleone where music concerts are organized and (c) street immersive augmented walking using HoloLens [7] in the City of Lucca (Sant' Andrea Street).

\subsection{Technoeconomic Scenarios}

The first service allows a complete immersive experience in an interactive environment. The already deployed services that offer low scale AR features though the use of smartphone screens, can be taken to the next level through the use of VR headgear, 
something that many museums are exploring as a service. In the frame of this use case, visitors are drawn into the experience by the addition of spatial audio or surround sound. While learning about the history of Puccini life they can also listen to the sound of his concerts and operas and textual infographics.

The $360^{\circ}$ live streaming service can be used in several applications allowing people to travel virtually to the location where an event (e.g. concert, interview etc.) is taking place. A technological challenge, in case of crowded events, the economic feasibility of this service is evaluated considering the case of Piazza Napoleone, located in the city centre of Lucca, during a live music concert [8]. The scenario consists of a $360^{\circ}$ camera, e.g. an Insta360 Pro device, placed near the stage live streaming the show to remote audience equipped with VR headsets. The concept is to provide an immersive experience for the end user and high engagement by placing the camera directly on the stage. The attendee of the event within the square can also use the service and make himself a part of the show rather than just part of the audience.

Finally, the cultural immersive street walking will allow the user to be involved in a wandering experience in Saint Andrew Street. Using rented AR headsets, the user can walk towards Guinigi Tower [9] can experience the full experience of augmented reality, getting additional content such as video description and the $3 \mathrm{D}$ reconstructed model of the tower itself. Other points of interest could be included in the future improving the service content and immersion wise.

\subsection{Technoeconomic Methodology}

The technoeconomic methodology used to assess telecom network investments and subsequently for the case of $5 \mathrm{G}$ has already been adopted in similar studies [10]. The core of a TE model is a database regularly updated with relevant data (e.g. network components) collected from the largest European telecommunication companies and vendors as well as benchmarks from the telecom market $[10,11]$ used to assess costs incurred to deploy and maintain a network as well as offer services.

The study period is always adapted to the case under investigation. For the deployment of $5 \mathrm{G}$ networks and considering the time needed for the technology to reach market maturity and pay back investments, an eight to ten-year period is adopted. A list of provided services and their market penetration over the study period are defined while econometric and price forecast models are used to define their tariffs. Services revenues can be calculated by vombining market penetration and tariff evolution.

To calculate the expenditures, the selected topology and dimensioning rules for radio and IT resources are used. Thus, alternative topologies can be easily incorporated in the TE analysis as long as the right input in terms of assets used is being provided. To calculate the required number of network components, demand forecasting is conducted using existing methodologies and market data. CAPEX is then calculated by combining the required number of components and their price for each year. A price evolution is calculated for all network components by using the extended learning curve model [12]. Maintenance costs consist of two parts: (i) the cost to repair, calculated as a fixed percentage of the total investments in network elements, and; (ii) the cost of repair work, using the mean time between failures (MTBF) and the mean time 
to repair (MMTR). Operating expenditures (OPEX) are also calculated in the tool, e.g. energy costs are evaluated based on the power consumption of components and the average cost of one $\mathrm{kWh}$ [13]. In the scenario investigated, network equipment is only used during the event and switched-off before and after.

By combining of all calculated costs and more general economic inputs (discount rate, cost of capital), the tool calculates the results necessary for Discounted Cash Flow analysis such as cash flows, Net Present Value (NPV), Internal Rate of Return (IRR), payback period and other notable economic figures.

\subsection{Demand Forecast}

The forecast is a very important part of the TE analysis since it helps quantify present and future costs and revenues. To estimate the $5 \mathrm{G}$ population penetration 6 steps were followed: (i) the definition of a $5 \mathrm{G}$ mobile subscription, (ii) understanding of regional deployment plans, (iii) understanding of qualitative factors affecting $5 \mathrm{G}$ adoption, (iv) observation of $3 \mathrm{G}$ and $4 \mathrm{G}$ adoption patterns, (v) estimation of $5 \mathrm{G}$ population penetration, and (vi) identification of risks that might hinder $5 \mathrm{G}$ adoption.

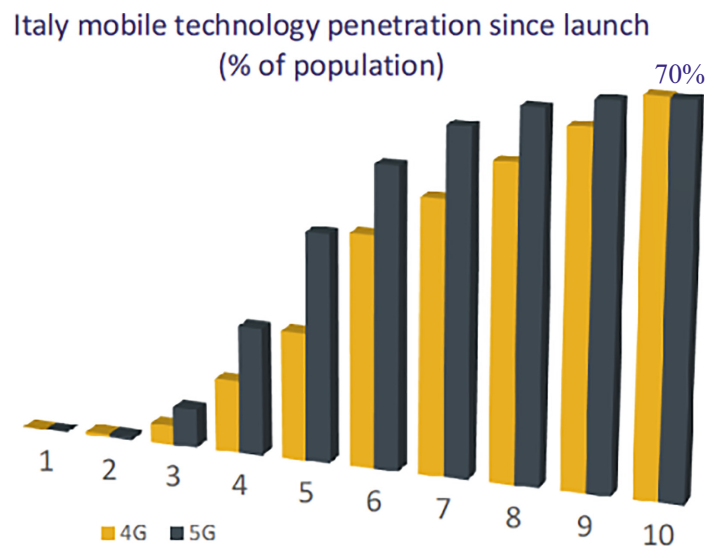

Fig. 1. $5 \mathrm{G}$ adoption curve

Following these steps, a global population adoption curve has been defined and shown in Fig. 1. Keep noted that machine-to-machine connections are not being considered for the purpose of this exercise.

\section{Results}

\subsection{Financial Results}

The financial results are what is to be studied and analyzed after the completion of TE model. During the study period, the NH invests in the necessary equipment in order to deploy the network including switches, servers, small cells etc. The length of fiber 
cables needed is also included using a geometric model. All individual cash flows as well as a cumulative cash flow line are presented in Fig. 2 for the underlying analysed network. The cumulative discounted cash flow, widely known as the cash balance, summarizes the total profit/loss at the end of each year of the business case.

From Fig. 2, it can be deduced that the balance is initially negative. This is somehow expected due to the high initial investments needed to deploy the network together and the relatively low demand for 5G services. As service demand increases, revenues grow at a faster pace than outflows, making the investment profitable within the study period. Specifically, our calculations show that the payback period for the investment is approximately 7 years with an IRR of $13 \%$ and a NPV of approximately $93 \mathrm{k} €$. Although initially CAPEX accounts for the bulk of the outflows, OPEX costs become more dominant towards the end of the study period.

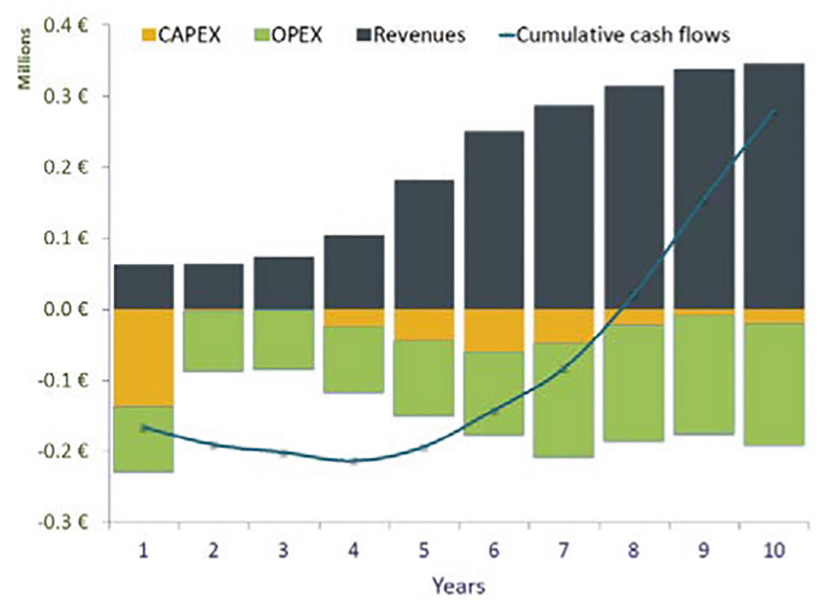

Fig. 2. Cash flows by year

\subsection{Sensitivity and Risk Analysis}

Having conducted the TE analysis, key outputs are the revenues, investments, lifecycle cost, cash balance, NPV and IRR, all important outputs that allow the evaluation of the investment analyzed leading to the next stage of understanding the most impactful parameters that surface within the TE model. To achieve this, sensitivity and risk analysis are being conducted allowing to take into consideration uncertainties involved. In the examined case, the parameters chosen for the sensitivity analysis were service tariffs, Napoleone piazza's capacity, CAPEX and the maximum number of HoloLens/Oculus devices at the end of the study period. Changed by $\pm 60 \%$ of their initial assumed values, the impact that each parameter has on the NVP is evaluated. As seen in Fig. 3, service tariffs have the biggest impact on NPV both positive and negative following their increase or decrease. Max number of headgear and CAPEX have similar impact on absolute terms while piazza capacity is not very impactful. It is worth noting that NPV's sensitivity to CAPEX will be diminished in the future along with advances in performance and capabilities of network components. 
A risk analysis follows to further analyze risky parameters that could influence the results of the use case. The tariffs of basic, 360, oculus and HoloLens services, 5G demand, CAPEX, piazza capacity and the maximum number of Oculus and HoloLens at the end of the study period were identified as the most impactful to the project. Modelling said variables using a probability density function that follows uncertainty assumptions that produce upper and lower limits, optimistic and pessimistic results all used as input into a ten thousand samples Monte Carlo simulation to output differentiated NPV results, useful in evaluating the project. The parameters showing the greatest influence on variance is the demand followed by tariff Oculus and Basic.

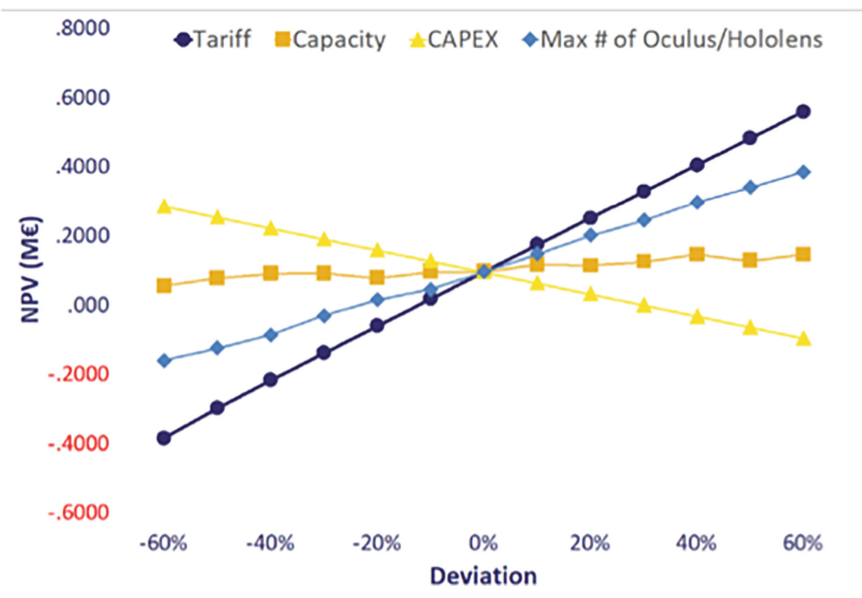

Fig. 3. Sensitivity analysis on the Net Present Value

\section{Conclusions}

In this paper, the business model of the Neutral Host was described, an entity providing wholesale $5 \mathrm{G}$ access in the form of end-to-end virtualized network slices along with a platform of functions and services able to support the individual providers. Due to the fact that $5 \mathrm{G}$ networks need much denser cell deployments, the 5GCity NH model, tested in Lucca and Sant Marti Superblock of Barcelona, seems as an enticing way to accelerate 5G networks' deployment. Additionally, the access to a service design toolkit opens up pathways for new revenue streams.

The model proposed is further examined through the technoeconomic analysis of a particular use case where MNOs in the City of Lucca is offering immersive video services using the NH network. The investment is breaking even in year seven of the study, due to an increase in revenues as the adoption of $5 \mathrm{G}$ brings along more end-users from year six and onwards, enough to counter increases in operational costs. As seen in the sensitivity analysis, demand will be a main contributing factor for the investment's viability as well as the ability to cut down on some of the costs that come along with improvements in small cell's technological capabilities. 
Acknowledgment. The research leading to these results has been supported by the EU funded H2020 5G-PPP project 5GCity (grant agreement No. 761508).

\section{References}

1. 1ITU-R Rec. M.2083-0: 2015IMT Vision - Framework and overall objectives of the future development of IMT for 2020 and beyond, September 2015

2. Colman-Meixner, C., et al.: Deploying a novel 5G-enabled architecture on city infrastructure for ultra-high definition and immersive media production and broadcasting. IEEE Trans. Broadcast. 65(2), 392-403 (2019). https://doi.org/10.1109/TBC.2019.2901387

3. Neokosmidis, I., Rokkas, T., Parker, M.C., Koczian, G., Walker, S.D., Escalona, E.: Assessment of socio-techno-economic factors affecting the market adoption and evolution of 5G networks: evidence from the 5G-PPP CHARISMA project. Telematics Inform. 34(5), 572 (2017)

4. Ksentini, A., Iqbal, M., Flinck, H.: Mobile edge computing potential in making cities smarter. IEEE Commun. Mag. 55(3), 38-43 (2017)

5. https://www.magicleap.com/

6. http://www.puccinimuseum.org/en/

7. https://www.microsoft.com/en-gb/HoloLens?icid=SSM_AS_Promo_Devices_HoloLens2

8. https://www.summer-festival.com/

9. http://www.turismo.lucca.it/en/luoghi-di-interesse/guinigi-tower

10. Monath, T., Kristian, N., Cadro, P., Katsianis, D., Varoutas, D.: Economics of fixed broadband access network strategies. IEEE Commun. Mag. 41(9), 132-139 (2003)

11. Katsianis, D., et al.: The financial perspective of the mobile networks in Europe. IEEE Pers. Commun. 8(6), 58-64 (2001)

12. Ims, L.A.: Broadband Access Networks. Springer, Boston (1998). https://doi.org/10.1007/ 978-1-4615-5795-1

13. Energy price statistics (2017). http://ec.europa.eu/eurostat/statistics-explained/index.php/ Energy_price_statistics 